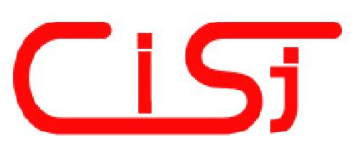

\title{
FRACTAL APPROACH TO ANALYSE THE INSTRUMENTAL TIME SERIES OF TEMPERATURE OBSERVATIONS
}

\author{
Yury Kolokolov ${ }^{1), 2)}$, Anna Monovskaya ${ }^{1), 2)}$ \\ 1) Russian Research Institute of Hydrometeorological Information - World Data Center, \\ Korolev str. 6, 249035, Obninsk, Russia, www.meteo.ru \\ ${ }^{2)}$ ITMO University, Kronverkskiy 49, 197101, St. Petersburg, Russia, www.ifmo.ru \\ 2kolo@mail.ru, anna.monovskaya@mail.ru
}

\begin{abstract}
The fractal approach allows to extend the knowledge about the nonlinear dynamics evolution that is caused by the growth in the capacity and adequacy of the information concerning regularities, uncertainties and abnormalities. The paper applies the fractal approach to analyze the instrumental time series observing the air temperature of the land surface during 1902-2012 years. An annual warming-cooling cycle is considered as a unit, so the peculiarities of the viewpoint on the temperature dynamics are connected with the analysis of the time series profiles. The corresponding fractal model - described the annual warming-cooling cycle dynamics - is adapted to the considered subject. Then it becomes possible to study comparatively the averaged, extremely and amplitude-frequency characteristics of the annual warming-cooling cycles. The observations - completed in the forty meteorological stations - are used in the six quasihomogeneous climate regions. The individual and comparative study of the local climate systems exhibits both overregional dominant tendencies and local uncertainties that enable to learn the local climate dynamics in more details. Copyright (C) Research Institute for Intelligent Computer Systems, 2015. All rights reserved.
\end{abstract}

Keywords: approach, time-series analysis, land surface air temperature observations, annual warming-cooling cycle, dominant tendency, local abnormality.

\section{INTRODUCTION}

The practical question how to analyse the climate dynamics in a certain geographic point for a season or few (up to three) years with high accuracy remains open till now $[1,2]$ because the annual warming-cooling cycle profile is supposed to be considered at least. What data and methods there are to solve the problem? The abilities of climate models to simulate the surface air temperature diminish when the coverage in time or place reduces [3]. The data of instrumental measurements seem to be the most reliable in this case, but the available instrumental data can be substantially restricted in size and resolution over time or/and space. In particular, the paleo-climate data allows to consider the surface air temperature evolution in timescales from hundreds years to millions years $[4$, 5], but the temperature variation within an annual warming-cooling cycle is not considered. The meteorological data contains the information concerning the temperature variation within an annual warming-cooling cycle, but the reliable meteorological data is, usually, about centenary long and no longer 300 years $[6,7]$.
To use the meteorological data it is necessary to find a compromise between methods of data acquisition and the coverage in time and space. The global mean surface temperature exhibits the substantial inter-annual variability, where the temperature trends based on short-term observations are sensitive to beginning and end dates, and do not show long-term climate tendencies [3]. The climate models show similar results: trend estimations can be significantly different in relation to the same region but several timescales $[8,9]$, or can be significantly different in relation to the same timescale but several regions $[10,11]$. In other words, the more space or timescale is considered in detail, the more number of exclusions in regional homogeneity can be founded. To analyse the dynamics of the annual warming-cooling cycles it is necessary to take into account the year-to-year variability of the cycles in frequency and amplitude characteristics $[8,10,12]$. Thus, the land surface air temperature observations should be considered as the nonlinear non-stationary noisy time series with complex profiles.

In this case the method of averaging can lead to distortions or losses of the actual information. 
Nevertheless the averaging and linear trends remain the most useful to show the dominant tendencies in dynamics of the surface air temperature [3]. How to skirt around the disadvantage of the averaging? One of the ways is proposed in the paper. Namely, the fractal approach $[13,14]$ to analyse the dynamics of the instrumental time series with the complicated profiles is applied to estimate the dynamics of both temperature extremums and amplitude-frequency characteristics of the annual warming-cooling cycles. The corresponding fractal model to describe the warming-cooling cycle dynamics is introduced in section 2. The fractal model is aimed to convert the initial temperature time series into the succession of the units, which are similar in general but can be different in dimensional modifications $[15,16]$. It allows to consider the dynamics of a local climate system in more detail. The results of the comparative analysis of the forty local climate systems are presented in section 3. Here the revealed overregional dominant tendencies and the reasonings on the peculiarity and abnormality of the local climate dynamics are summarized. Finally, conclusion is presented.

\section{FRACTAL VIEWPOINT ON ANNUAL WARMING-COOLING CYCLE}

Let us consider the short-term warming-cooling cycles made by the instrumental measurements that are similar to the long-term warming-cooling cycles based on paleo-climate data. Then the notion of an annual warming-cooling cycle (Fig. 1(a)) means a part of time series between the successive temperature minimums (hereafter $T_{\min }$ ), where the time series consists of the land surface air temperature observations averaged per day (hereafter T). Let us consider the peculiarities of the annual warming-cooling cycles from the following practical point: how to estimate when and what temperature extremums (hereaftre $T_{\min }$ and $T_{\max }$ ) will occur in the nearest future (Fig. 1(a))?

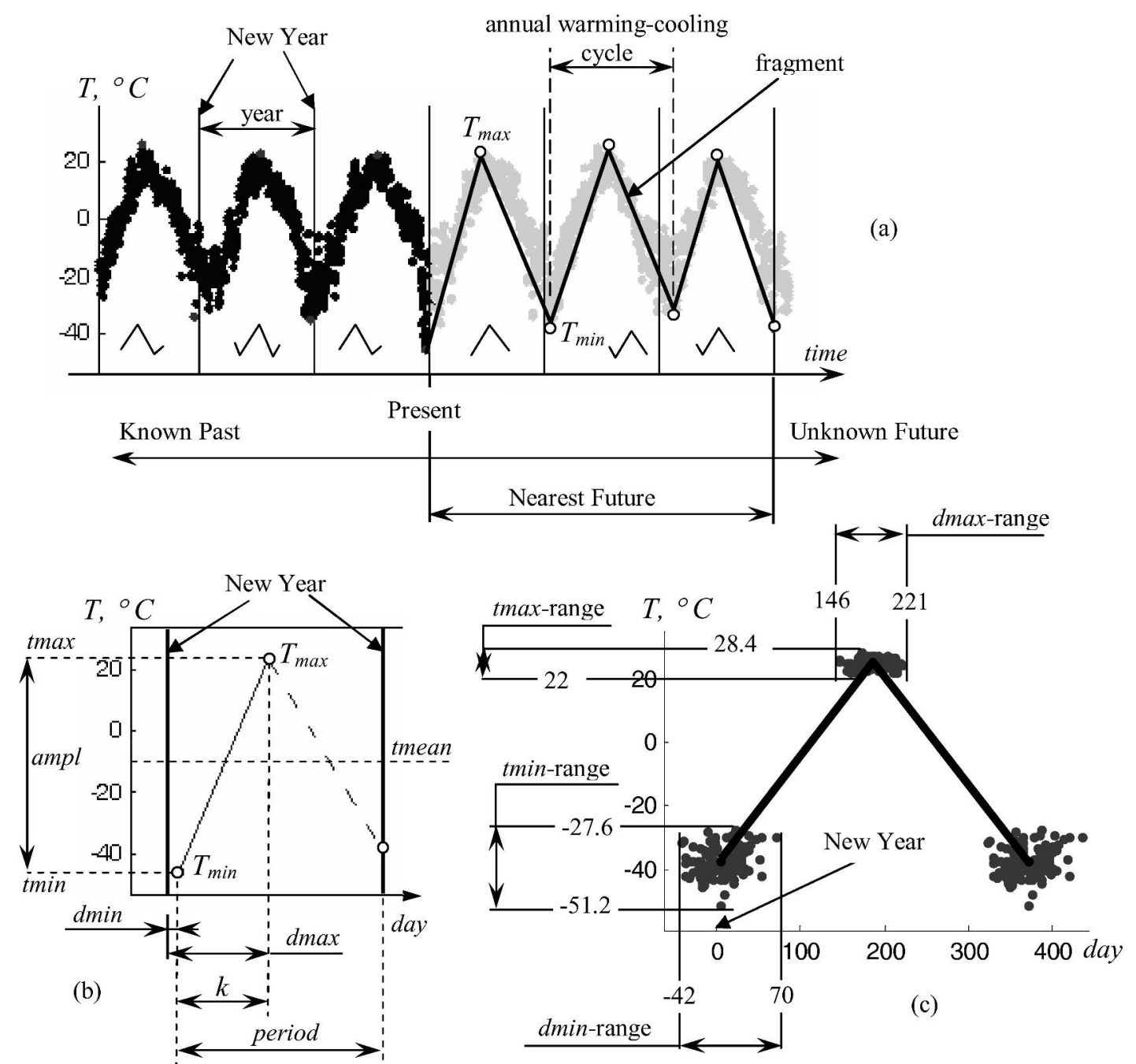

Fig. 1 - A part of $T$-time series to illustrate the notion of annual warming-cooling cycle (a); characteristics of an annual warming-cooling cycle (b); the ranges of the temperature extremums in Tomsk (WMO-index 29430) over

1902-2012 years (c). The historical meteorological data here and after were provided by Russian Research Institute of Hydrometeorological Information - World Data Center, www.meteo.ru. 
Let dmin and dmax be the time coordinates of $T_{\min }$ and $T_{\max }$ correspondingly; let tmin and tmax be the temperature coordinates of $T_{\min }$ and $T_{\max }$ correspondingly (white points in Fig. 1(b)). Then $T$-time series can be considered as the alternations of the extremums, where the triangle-like units are formed between the successive $T_{m i n}$-values (solid lines in Fig. 1(a)). Such process was denoted as the time series fragmentation [15], where each fragment represents a geometrical invariant with the fractal properties $[13,16]$. At the same time, each fragment represents the linearized annual warming-cooling cycle. Then the model of $T$-time series based on the fragmentation is hereafter denoted as a fractal model. The fractal model will be described hereafter by the following characteristics of each annual warming-cooling cycle (Fig. 1(b)): the time and temperature coordinates of the extremums (dmin, $d$ max and tmin, tmax); the averaged temperature (tmean), amplitude ( $\mathrm{ampl}$ ) and duration (period) in relation to the corresponding fragment; the relative duration of the warming stage $(k)$.

Why it is necessary to find other models in addition and/or instead of the traditional one? Climate dynamics is analyzed in terms of the phase variables (first of all, temperature) averaged by year or more, but the consensus of opinions on the averaging is absent even today $[8,12,17]$ : average over what and in what way? For example, the decadal timescale is considered as one of the most promising $[1,2]$. Both 10-year and 20-year windows in relation to the reference period 1986-2005 years are used in the recent report of Intergovernmental Panel of Climate Change [3]. At the same time, the climate dynamics remains to be estimated by 30 -year windows in relation to the reference period 1961-1990 years according to the current meteorological convention [17, 18]. The average concerns the notion of one year rather then the notion of the annual warming-cooling cycle. So, the annual dynamics of a local climate system is usually described by the temperature variation averaged per month. Correspondingly, the annual temperature variation maps traditionally 12 states of a local climate system between the successive minimums in January (for the Nothern hemisphere), for example $[12,19,20]$.

However the frequency and amplitude characteristics of annual warming-cooling cycles can be essentially variable $[8,10,12]$. In particular, $d m i n$-variation leads to that, that $T_{m i n}$-values can be observed beyond January. For example, dmin-range can be from the November 19 to the March 11 (Fig. 1(c)). If the border of an averaging window is fixed (for example, the $1^{\text {st }}$ of January), then a particular $T_{m i n}$-value can miss the averaging window (for example, the first and fourth fragments in
Fig. 1(a)). Or two $T_{m i n}$-values can hit the same averaging window (for example, the third fragment in Fig. 1(a)). So, the ampl- and tmean-values identified in a certain year will be different from the actual ones within the corresponding annual warming-cooling cycle. Such cases are not unique over last 111 years, and can occur several times successively. In particular, the range of periodvalues can be estimated from $75 \%$ to $125 \%$ of one year, and the range of ampl-values can be estimated from $66 \%$ to $100 \%$ of the maximal observable amplitude for example in Tomsk (Fig. 1(c)). Generally, the chaotic-like variation of the characteristics of the annual warming-cooling cycles in wide ranges seems to be natural over last hundred years at least. For example, the ranges of tmax-, tmin-, dmax-, dmin-values are illustrated in Fig. 1(c), where the dmin-range is about $30 \%$ of year and the dmax - range is about $20 \%$ of year. Similar results regarding 1902-2012 years can be illustrated for all the forty meteorological stations considered in the paper.

As a result, the fixed 1-year window can distort the description of annual temperature tendencies. For example, let us consider tmean- and dmin-time series (Fig. 2) to illustrate the differences of the results calculated per year and per fragment.

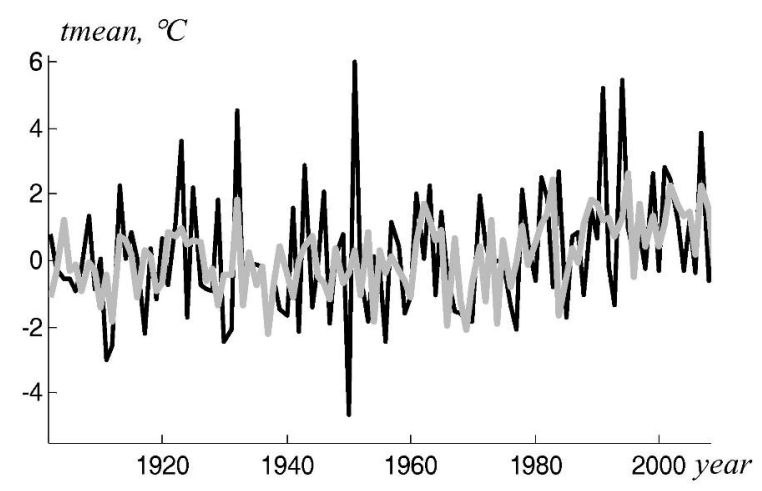

dmin, day in relation

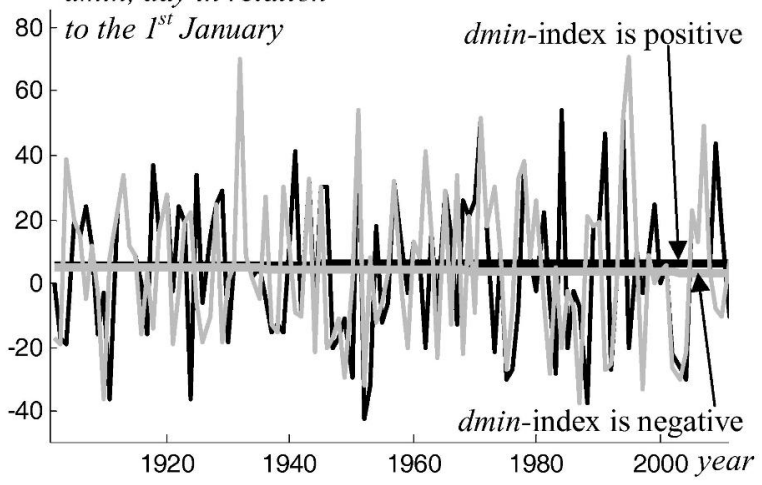

time series calculated per year; time series calculated per fragment.

Fig. 2 - Examples of tmean-and dmin-time series (a,b correspondingly) calculated per year and per fragment in Tomsk over 1991-2012 years. 
The profiles of tmean-time series calculated per year are smoother than the ones calculated per fragment; correspondingly, there are the distortions of the real extremums at least (Fig. 2(a)); dmintrends calculated per year and fragment can be contrary in sign (Fig. 2(b)), correspondingly, the forecasts can be quite different. If it is necessary to analyse the dynamics of more than one local climate system, then it is necessary to choose a common averaging window to keep the correctness of the comparative analysis. For example, in the case of six meteorological stations the averaging windows should be chosen from the 1 May to the 1 October. Strictly speaking, the elements formed by 1-year window remain heterogeneous in shape to a greater or lesser extent. For example, the sketches of 1-year elements are illustrated in Fig. 1(a). From the mentioned viewpoint the fractal model seems to be more proper to describe the observed dynamics.

\section{OBTAINED RESULTS}

Let us use the fractal model to analyse forty meteorological stations located in six quasihomogeneous climatic regions of East Europe, Siberia and Far East. The temperature measurements made at these stations are uninterrupted over the same timescale of 111 years long. The historical meteorological data were provided by Russian Research Institute of Hydrometeorological Information - World Data Center. The dynamics of each local climate system is analyzed by the following way: the characteristics of the annual warming-cooling cycles are determined for each fragment; the evolution of each characteristic is presented as one of the constituents of the local climate dynamics; the linear trend coefficient (hereafter index) is determined for each constituent. So, each $T$-time series is presented as the ensemble composed of the seven constituents (dmin-, dmaxtmin-, tmax-, ampl-, tmean-, $k$-time series), which are estimated by the corresponding dmin-, dmaxtmin-, tmax-, ampl-, tmean-, $k$-indexes. The ensemble viewpoint exhibits potentially more particularities concerning the evolution of time series $[14,21]$.

What it means in relation to temperature time series? Let us consider several examples. In particular, the following over-regional dominant tendencies were revealed on the basis of the index combinations (hereafter index scheme): the increase in the averaged temperature (i.e. the positive tmeanindex) is realized with the decrease in the temperature amplitude (i.e. the negative amplindex), the increase in the temperature minimums (i.e. the positive tmin-index), and the decrease in temperature maximums (i.e. the negative tmaxindex). And the moments of temperature minimums and maximums become to be later, i.e. the positive dmin- and dmax-indexes take place. The corresponding index scheme is illustrated in Fig. 3(a).

But it is just one of the index schemes. Mentioned combination of the temperature indexes can be realized with the negative dmin- and dmaxindexes in, for example, Eniseyisk (WMO-index 29263). It is interesting that different index schemes can be observed not only within the same quasihomogeneous climatic region, but also for the stations at a quite short distance. Each of the considered climatic regions seems to be rather heterogeneous from the viewpoint of the warmingcooling cycle dynamics. It confirms the known heterogeneity in short-term timescales regarding the temperature trends in North America shown in [11], regarding the rainfall extremes in West Europe shown in [22], as well as regarding the opposite temperature tendencies in timescales of ten thousands years [23]. The fractal model reveals that annual warming-cooling cycles can demonstrate not only more rapid rise in temperature, but also more rapid fall in temperature. It confirms the conclusion in [10], that annual warming-cooling cycles are characterized by variable periodicity in short-term timescales. But it is interesting, that similar variability of warming-cooling cycles is typical in more long timescales of ten thousands years $[4,5]$.

How the fractal analysis can be used differently? Let us continue to consider the local dynamics in Tomsk, and analyse the index scheme in Fig. 3(a). First, it allows to limit the ranges of the annual variation: the negative tmax-index means that the largest temperature maximum is no more than $M_{\text {tmax }}+3 \sigma_{\text {tmax }}$ (L1-line in Fig. 3(b)), where $M$ is a mathematical expectation, $\sigma$ is a root-mean-square deviation, the subscript denotes the corresponding constituents; the positive tmin-index means that the temperature minimum is no less than $M_{\text {tmin }}-3 \sigma_{\text {tmin }}$ (L2-line in Fig. 3(b)). The positive $d m i n$-index allows to limit the earliest moment of the temperature minimum ( $L 3$-line $M_{d \min }-3 \sigma_{d m i n}$ in Fig. 3(b)), and the positive dmax-index allows to limit the earliest moment of the temperature maximum (L4-line $M_{d \max }+4 \sigma_{d \max }$ in Fig. 3(b)). The latest moments of both temperature minimum and maximum are more uncertain: regarding the nearest future it is accepted only approximately as $M_{d \min }+4 \sigma_{d \min }$ and $M_{d \max }+4 \sigma_{d \max }$, correspondingly (dashed L5-, L4- lines in Fig. 3(b)). 


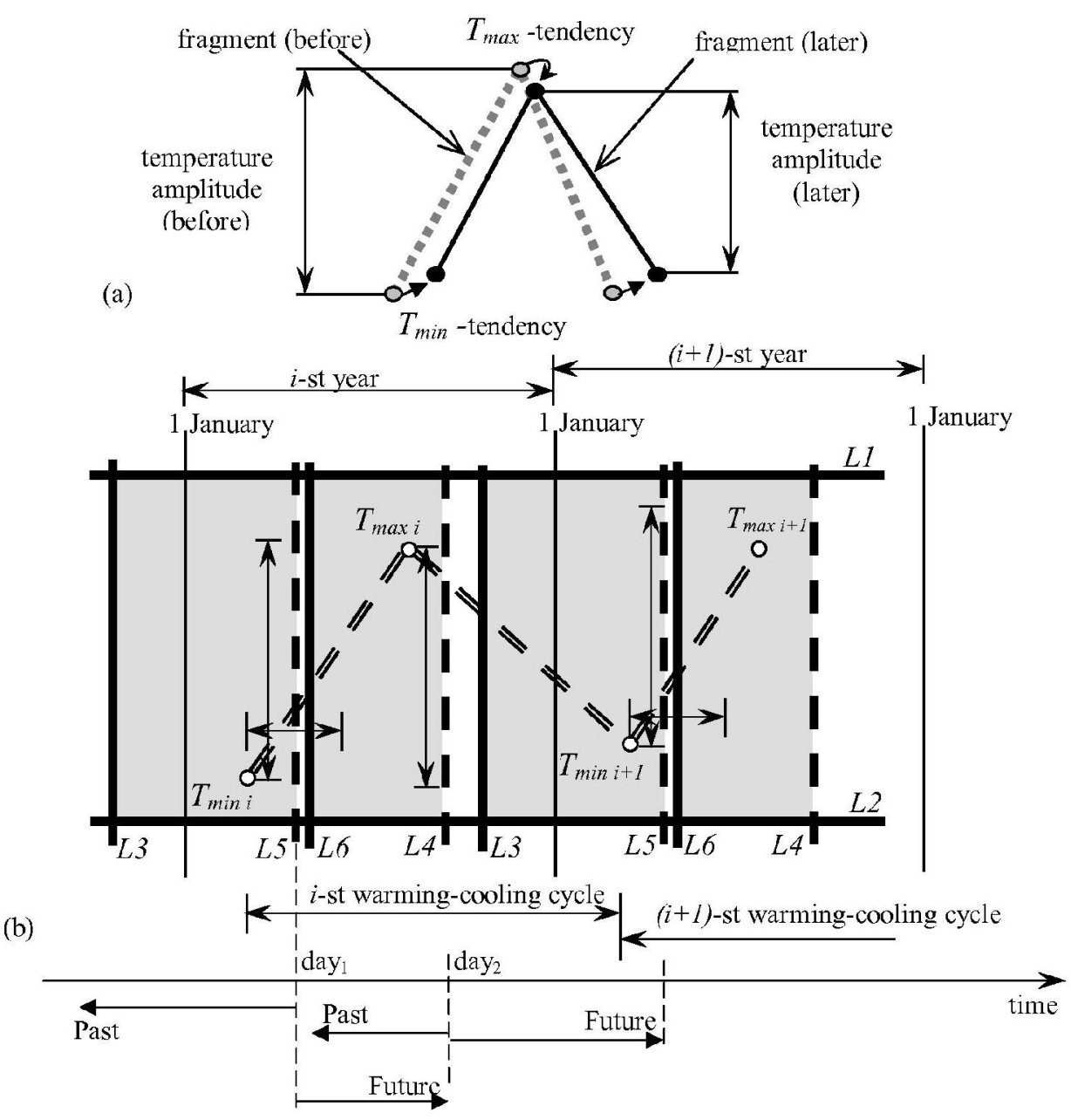

Fig. 3 - Index schemes to summarize the pattern of the dominant tendencies in Tomsk over 1902-2012 years (a) and an example of the corresponding consequences from such local evolution (b).

Second, the characteristics allow to specify the limits of the acting dynamics and indicate the potential possibilities to forecast. In particular, the negative ampl-index means that the temperature difference between the neighboring extremums is no more than $M_{\text {ampl }}+3 \sigma_{\text {ampl }}$ (the vertical two-sided arrows in Fig. 3(b). The positive $k$-index means that the duration between the minimum and maximum is no less than $M_{k}+3 \sigma_{k}$ days (the horizontal two-sided arrows in Fig. 3(b)). So, as soon as $L 5$-line is crossed over (Fig. 3(b), at the moment $d a y_{1}$ ), it becomes possible to make more certain the characteristics of the temperature maximum $\left(T_{\max }\right)$ in value and time within the running warming-cooling cycle: no more than $\operatorname{tmin}_{i}+\left(M_{\text {ampl }}+3 \sigma_{\text {ampl }}\right)$ and no earlier than L6-line. As soon as L4-line is crossed over (Fig. 3 (b), at the moment $\left.d a y_{2}\right)$, it becomes possible to make more certain characteristics of the temperature minimum $\left(T_{\min \mathrm{i}+1}\right)$ in value and time within the next warming-cooling cycle: no less than $\operatorname{tmax}_{i}-\left(M_{\text {ampl }}+3 \sigma_{\text {ampl }}\right)$, and no later than L5-line, and so on cycle-by-cycle.

Third, the substantial exceeding the dmin-range over dmax-range as well as tmin-range over tmax- range (Fig. 1(c)) leads to the following: the majority of unfavourable and damage abnormalities (96\%) should be typical for the phenomena towards coldness. And it is confirmed by the observable data (Fig. 4).

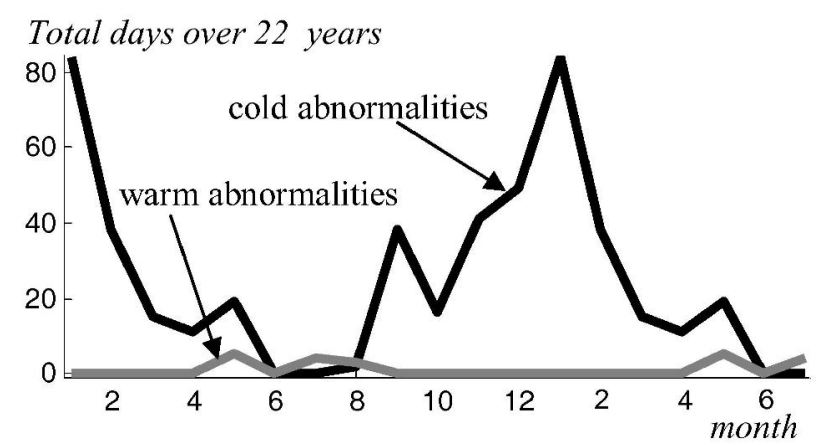

Fig. 4 - Unfavourable and damage abnormalities observed in Tomsk over 1991-2012 years.

Generally speaking, the introduced index schemes allow to estimate the potential abilities to forecast the local annual temperature evolution from the viewpoint where the more or less uncertainty in dynamics takes place at least. For example, the 
index scheme in Fig. 3(b) exhibits that it is more complicated to make forecasts in Spring (the narrow white gaps in Fig. 3(b)), then in Autumn (the bold white gap in Fig. 3(b)). It confirms both the complexity of forecasts in 1-year timescale [2] and regional differences in the forecast probability [24].

\section{CONCLUSION}

The local temperature evolution can be so much heterogeneous, that traditional estimations can loss important information. One of the promising directions to develop the traditional estimations is connected with the fractal approach, which allows to extend the knowledge concerning regularities, uncertainties and abnormalities in application to the annual warming-cooling cycle dynamics. In this case the temperature observations are presented as the homogeneous objects (so-called fragments). Thus, the ensemble of traditional, rare considered and unconsidered characteristics of an annual warmingcooling cycle can be used to describe the annual evolution. It allows to exhibit the dominant patterns, in accordance to which the behavior of a local climate system is realized. In particular, it is revealed three main patterns which are observed in more than $70 \%$ of all the cases. It is interesting the distribution among these cases: one of the patterns (the normal pattern) is observed in more than 50\% of cases; each of the rest two patterns (the auxiliary patterns) is observed in $25 \%$ of cases. The normal pattern is typical for two climatic domains which locate extremely far from each other (the Central European part and the Far East), and the auxiliary patterns are observed between these domains. So, there are, at least, three regions with quite well distinguished and qualitatively different dominants in evolution of the local dynamics. It means that the homogeneity of the dynamics within the regional climatic domains can be rather questionable from the viewpoint of the warming-cooling cycle evolution. At present there are not the established models to explain such facts $[3,4,5,23]$. Nevertheless, the alternations between the patterns look like rather the behavior under the intermittency phenomena [25]. The main disadvantage of the fractal approach is connected with the comparatively labour data processing, since there are some unformalized analytical procedures with expert opinions.

\section{ACKNOWLEDGMENT}

We thank Dr. O.N. Bulygina, Dr. V.M. Veselov and S.I. Shamin for providing easy access to the historical data of RIHMI-WDC (www.meteo.ru). The work is partially financially supported by Government of Russian Federation (Grant 074-U01) and by Ministry of Education and Science of Russian Federation (Project 14.Z50.31.0031).

\section{REFERENCES}

[1] M. Hoerling, J. W. Hurrell, A. Kumar, L. Terray, J. Eischeid, P. Pegion, T. Zhang, $\mathrm{X}$. Quan and T. Xu, On North American decadal climate for 2011-20', Journal of Climate, (24) (2011), pp. 4519-4528.

[2] G. J. Boer, V. V. Kharin and W. J. Merryfield, Decadal predictability and forecast skill, Climate Dynamics, (41) (2013), pp. 1817-1833.

[3] Climate Change 2013: The Physical Science Basis. Contribution of Working Group I to the Fifth Assessment Report of the Intergovernmental Panel on Climate Change [T. F. Stocker, D. Qin, G.-K. Plattner, M. Tignor, S. K. Allen, J. Boschung, A. Nauels, Y. Xia, V. Bex, P. M. Midgley (eds.)]. Cambridge University Press, Cambridge, United Kingdom and New York, NY, USA.

[4] J. A. Rial, R.A. Pielke, M. Beniston, M. Claussen, J. Canadell, P. Cox, H. Held, N. de Noblet-Ducoudre, R. Prinn, J. F. Reynolds and J. D. Salas, Nonlinearities, feedbacks and critical thresholds within the Earth's climate system, Climatic Change, (65) (2004), pp. 1-38.

[5] J. M. Thompson and J. Sieber, Predicting climate tipping as a noisy bifurcation: a review, International Journal of Bifurcation and Chaos, (21) 2 (2011), pp. 399-423.

[6] B. Todd, N. Macdonald, R. C. Chiverrell, C. Caminade and J. M. Hooke, Severity, duration and frequency of drought in SE England from 1697 to 2011, Climatic Change, (121) (2013), pp. 673-687.

[7] T. J. Porter, M. F. J. Pisaric, R. D. Field, S. V. Kokelj, Th. W. D. Edwards, P. de Montigny, R. Healy and A. N. LeGrande, Spring-summer temperatures since AD 1780 reconstructed from stable oxygen isotope rations in white spruce tree-rings from the Mackenzie Delta, northwestern Canada, Climate Dynamics, (42) (2014), pp. 771-785.

[8] Zh. Wu, E. K. Schneider, B. P. Kirtman, E. S. Sarachik, N. Huang and C. J. Tucher, The modulated annual cycle: an alternative reference frame for climate anomalies, Climate Dynamics, (31) (2008), pp. 823-841.

[9] B. Christiansen, Changes in temperature records and extremes: Are they statistically significant?, Journal of Climate, (26) (2013), pp. 7863-7875.

[10] X. Chen, Yu. Zhang, M. Zhang, Y. Feng, $\mathrm{Zh} . \mathrm{Wu}, \quad \mathrm{F}$. Qiao and N. Huang, Intercomparison between observed and 
simulated variability in global ocean heat content using empirical mode decomposition, part I: modulated annual cycle, Climate Dynamics, (41) (2013), pp. 2797-2815.

[11] J. C. Rogers, The $20^{\text {th }}$ century cooling trend over the southeastern United States, Climate Dynamics, (40) (2013), pp. 341-352.

[12] S. Pezzulli, D. B. Stephenson and A. Hannachi, The variability of seasonality, Journal of Climate, (8) (2005), pp. 71-88.

[13] Yu. Kolokolov and A. Monovskaya, Fractal approach, bifurcation poker and SUC-logic for nonlinear dynamics forecasting, International Journal of Bifurcation and Chaos, (23) 12 (2013), pp. 1350201.

[14] Yu. Kolokolov and A. Monovskaya, Estimating the uncertainty of the behavior of a PWM power converter by analyzing a set of experimental bifurcation diagrams, International Journal of Bifurcation and Chaos, (23) 4 (2013), pp. 1350063.

[15] Yu. Kolokolov and A. Monovskaya, From geometric invariants and symbolical matrixes towards new perspectives on forecasting of PWM converter dynamics, Chaos, Solitons \& Fractals, (42) 3 (2009), pp. 1868-1877.

[16] Yu. Kolokolov and A. Monovskaya, Computational researches: reasoning on evolution of technical and biological systems, Proceedings of the $7^{\text {th }}$ IEEE International Conference on Intelligent Data Acquisition and Advanced Computing Systems: Technology and Applications, 12-14 September, 2013, Berlin, Germany, pp.801-806.

[17] J. Sillmann, V. V. Kharin, F. G. Zwiers and $\mathrm{X}$. Zhang, Climate extreme indices in the CMIP5 multi-model 57 ensemble. Part 2: Future climate projections, Journal of Geophysical Research, (118) (2013), pp. 1-21.

[18] Ch. Essex, Climate theory versus a theory for climate, International Journal of Bifurcation and Chaos, (21) 12 (2011), pp. 3477-3487.

[19] S. Fatichi, V. Y. Ivanov and E. Caporali, Assestment of a stochastic downscaling methodology in generating an ensemble of hourly future climate time series, Climate Dynamics, (40) (2013), pp. 1841-1861.

[20] L. Menut, O. P. Tripathi, A. Colette, R. Vautard, E. Flaounas and B. Bessagnet, Evaluation of regional climate simulations for air quality modeling purposes, Climate Dynamics, (40) (2013), pp. 2515-2533.

[21] Yu. Kolokolov and A. Monovskaya, Preventive diagnosis of pulse power converter dynamics, Automation and Remote Control, (70) 7 (2009), pp. 1228-1242.

[22] P. Willems, Multidecadal oscillatory behaviour of rainfall extremes in Europe, Climatic Change, (120) (2013), pp. 931-944.
[23] X. Yang, J. A. Rial and E. P. Reischmann, On the bipolar origin of Heinrich events, Geophysical Research Letters, (41) (2014), pp. 9080-9086.

[24] S. Corti, A. Weisheimer, T. N. Palmer, F. J. Doblas-Reyes and L. Magnusson, Reliability of decadal predictions, Geophysical Research Letters, (39) (2012), L21712.

[25] Yu. Kolokolov and A. Monovskaya, From modifications of experimental bifurcation diagrams to operating process stability margin, International Journal of Bifurcation and Chaos, (23), 7 (2013), pp. 1330024.

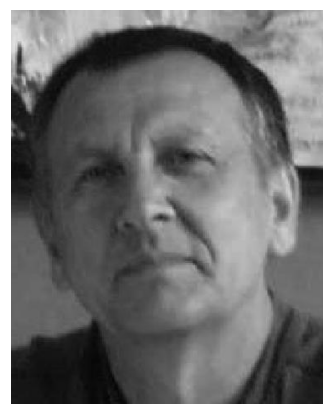

Yury Kolokolov received the M.S. degree in 1972 (Tomsk Politechnical Institute, Russia), PhD degree in 1978 (Tomsk Politechnical Institute, Russia), Russian academic degree of Senior Doctor in 1990 (Tomsk University of Control and Radioelectronics, Russia). From 1993 to 2007 he worked as a professor and Head of the Dept. of Design and technology of electronic systems (State Technical University of Orel, Russia), from 2007 to 2012 he worked as a professor and Director of Institute of Control Systems and Information Technologies (UGRA State University, Khanty-Mansiysk, Russia). He is currently a chief researcher (Russian Research Institute of Hydrometeorological Information - World Data Center, Obinsk, Russia and ITMO University, St. Petersburg, Russia). His research interests include theories and practice of control and stability, nonlinear dynamics and catastrophes regarding complex technical, natural and social systems.

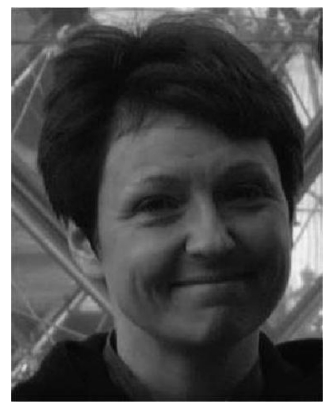

Anna Monovskaya received the M.S. degree in 2001 (State Technical University of Orel, Russia), PhD degree in 2005 (State Technical University of Orel, Russia and University of Technology of Troyes, France), Russian academic degree of Senior Doctor in 2010 (UGRA State University, KhantyMansiysk, Russia). From 2007 to 2012 she worked as a professor and Head of the Dept. of Automated systems of data processing and control (UGRA State University, Khanty-Mansiysk, Russia). She is currently a chief researcher (Russian Research Institute of Hydrometeorological Information - World Data Center, Obinsk, Russia and ITMO University, St. Petersburg, Russia). Her research interests include theories and practice of control and stability, nonlinear dynamics and catastrophes regarding complex technical, natural and social systems. 